\title{
Blotch Detection for Film Restoration
}

\author{
Alvaro Pardo* \\ School of Engineering and Technologies \\ Universidad Catolica del Uruguay \\ apardo@ucu.edu.uy
}

\begin{abstract}
Blotches are one of the most common film degradations that must be detected and corrected in the process of film restoration. In this work we will address the problem of blotch detection in the context of digital film restoration. Although there are several methods for blotch detection, in the literature their evaluation is usually subjective. In this work we propose a new method for blotch detection and an objective methodology to evaluate its performance. We show that the proposed method outperforms other existing methods while using this objective metric.
\end{abstract}

\section{Introduction}

Digital Film Restoration is a relevant problem that has attracted the attention of the digital image processing community. Due to aging, films undergo several degradation processes, some natural and others linked to poor storing conditions or careless manipulation. The principal defects in degraded films are: dirt-dust (noise), scratches, blotches, lost or degradation of color, film grain, missing frames, etc. For details we refer to [4] and [6].

In this work we will address the problem of blotch detection. Blotches are one of the most common film degradations. They are caused by the loss of film covering (white blotches) or dirt covering (dark blotches) the surface of the film. They are localized in a frame and produce temporal discontinuities. As we will see later, blotches can be modeled as a random process.

The main goals of this paper are the following. Firstly, we propose a blotch detection method that outperforms the previous ones using an objective methodology here proposed. Second, we also propose an objective methodology to evaluate the performance of blotch detection methods. The evaluation of our proposed method will be done against a traditional method [7] and a recently proposed one 2].

The outline of the paper is as follows. In Section 2 we review the related methods in the literature and concentrate ourselves in the two methods that will be evaluated and compared with our approach. Then in Section 3 we present our proposal for blotch detection. In Section 4 we discuss the evaluation setting and present the obtained results and finally in Section 5 we conclude and discuss the results.

\footnotetext{
* This work was soported by STIC-AMSUD MMVPSCV-Mathematical Models for Visual Perception ans Subpixel Computer Vision and ANII-FMV-2009-1-3042. 


\section{Related Methods}

Since blotches are localized temporal discontinuities most existing methods in the literature rely on motion compensation and frame differencing to detect pixels with large differences. Although most existing algorithms propose motion compensation without further consideration, it must be noted that motion compensation in noisy sequences is not an easy task. In this work we will assume that motion estimation and compensation can be performed. As we will see later, in our proposal we specially consider the fact that motion estimation can fail.

SROD 7] is a classical method for blotch detection which is very attractive for its simplicity and efficiency. Given the current frame, $F_{n}(x, y)$, the method computes pixel-wise differences with previous and next motion compensated frames, $F_{n-1}^{c}(x, y)$ and $F_{n+1}^{c}(x, y)$ respectively. Let $\left(r_{1}, r_{2}, r_{3}\right)$ be the pixel values $\left(F_{n-1}^{c}(x, y-1), F_{n-1}^{c}(x, y), F_{n-1}^{c}(x, y+1)\right)$. Similarly $\left(r_{4}, r_{5}, r_{6}\right)$ are the pixel values in a column centered at $F_{n+1}^{c}(x, y)$. The SROD declares the pixel $(x, y)$ as part of a blotch if:

$$
F_{n}(x, y)-\max \left\{r_{1}, \ldots, r_{6}\right\}>T \text { or } F_{n}(x, y)-\min \left\{r_{1}, \ldots, r_{6}\right\}<-T,
$$

where $T$ is the detection threshold of the algorithm.

In [2] the authors proposed a blotch detection method that follows the a contrario methodology [3]. Not only they present a method to detect the blotches candidates but also they embed in the same method a validation step. The Adaptive Spike Detection Index (ASDI) is defined as:

$$
A S D I(x, y)=\max \left\{F x\left(D x^{-}\right), F x\left(D x^{+}\right)\right\} \operatorname{sign}\left(D x^{+} . D x^{-}\right),
$$

where $D x^{+}$and $D x^{-}$are the average of pixel differences between the current frame and previous and next motion compensated ones across a neighborhood $N$ centered at $(x, y) . F x(q)$ is the tail of the Gaussian distribution, $\mathcal{N}\left(0, \sigma_{x}^{2} /|N|\right)$, $|N|$ is the area of the neighborhood $N$ and $\sigma_{x}$ is the local standard deviation of the frame differences. For the simulations $N$ is set as in $[2]$ as a $3 \times 3$ neighborhood.

A pixel $(x, y)$ is validated as a blotch if $0 \leq A S D I(x, y) \leq \epsilon /|\Omega|$, where $|\Omega|$ is the size of the frame.

\section{Proposed Method}

As we already mentioned, motion compensation is a challenging task in the case of noisy sequences. For degraded films the problem can be even worst due to the blotches themselves, the scratches, film grain, etc. For this reason in our method, after motion estimation and compensation, we allow some local adaptation during blotch detection.

Given the pixel $(x, y)$ we consider a search window $W$ centered at this pixel to define the proposed detection as:

$$
D(x, y)=\min \left\{\left|F_{n}(x, y)-F_{n-1}^{c}\left(x^{\prime}, y^{\prime}\right)\right|,\left|F_{n}(x, y)-F_{n+1}^{c}\left(x^{\prime}, y^{\prime}\right)\right| /\left(x^{\prime}, y^{\prime}\right) \in W\right\} .
$$


The underlying idea behind this definition is to look for the most similar pixel in an extended search window $W$. In this way we can cope with errors in the steps of motion estimation and compensation. Although we use pixel-wise differences the idea can be extended to compute the differences using neighborhoods around each pixel, i.e. block based distances. This is in fact the idea applied in [2].

In order to detect the blotch candidates we threshold $D(x, y)$ and compute $B(x, y)=D(x, y)>T d$. We choose a simple threshold $T d \geq 1$ and complement the detection with a validation step. Therefore, in the thresholding step we over detect to then, in the second step, validate the detections.

Our validation step is similar as the one proposed in [2] due to its probabilistic nature. However, in contrast with the validation step in [2] we validate blotches candidates and not pixels. For every connected component in the image $B(x, y)$ we compute its probability given the empirical distribution of the values in $D(x, y)$.

For every connected component $C$ of $B(x, y)$ we compute the minimum of $D(x, y)$ for pixels in $C$. Let's call this value

$$
\text { dmin }=\min \{D(x, y) /(x, y) \in C\} .
$$

Then, using the empirical distribution of values in $D(x, y)$ we define

$$
p=\operatorname{Probability}(D(x, y)>d m i n) .
$$

Assuming that pixels are independent 1 we define the probability of observing the connected component $C$ as:

$$
\operatorname{Probability}(C)=p^{|C|}
$$

where $|C|$ is the area of the connected component $C$.

We declare a blotch as valid if its probability of occurrence, given the background model obtained as the empirical distribution of values of $D(x, y)$, is very small. To do that we use a logarithmic scale and declare a connected component $C$ as a blotch if $\log (\operatorname{Probability}(C))=|C| \log p<p *$. In all the experiments we set $p *=-100$.

The previous validation step favors large connected components and small well contrasted ones. In the first case, since the size of the connected component $C$ is used as an exponent, the larger $C$ the smaller the probability. On the other hand, if the $C$ is well defined, i.e. large differences $D(x, y)$, its probability is also small. Both cases are the most distinguishable types of blotches as they strike from spatial and temporal neighboring pixels.

It is important to note that the only parameter in our method is the threshold, $p *$, used to validate the connected components.

\section{Evaluation}

Although there exists an extensive literature about blotch detection the evaluation of the different proposals is usually performed in a subjective manner or

\footnotetext{
${ }^{1}$ This is in some way a naive assumption.
} 
using a limited number of labeled frames used as ground truth. This makes it very difficult to compare different methods in an objective way. Hence we propose a method to simulate blotches and a set of scores to compare the performance of each method.

\subsection{Blotch Simulation}

As stated in the introduction blotches are localized regions of temporal discontinuity. The size and shape of these regions can vary and the probability of observing a blotch in one frame does not constrain the same the observation in other frames. Based on these considerations we devised a simple method to artificially create blotches.

We start generating an image with Gaussian noise of zero mean and variance $\sigma$. Then we filter this image with an box filter of size $5 \times 5$ and then threshold it at $\sigma / 4$. The result is an image with some connected components of pixels with varying size and shape. Now we have to simulate the random nature of the blotch observation and its random intensity. For that we first randomly select the blotches using an uniform distribution and then randomly select the grey level of the blotch in [gmin, gmax]. We simulate only dark blotches but the generation of bright ones is straight forward. Finally, once we have the image of blotches we filter with a Gaussian window to smooth its borders. In Figure 4.3 we show a sample realization of this proposal.

\subsection{Evaluation Scores}

For the objective evaluation we propose two set of scores; one set to measure the performance of the detection at the pixel level and the other one at the blotch level. In all cases we assume to know the number of blotches and their positions in the frame as a result of the simulation process described in previous section.

We define the following scores:

- FNp:= Number of False Negative pixels,

- FPp:= Number of False Positive pixels,

- FN:= Number of False Negative Blotches,

- FP:= Number of False Positive Blotches,

$-\mathrm{P}:=$ Precision at pixel level,

$-\mathrm{R}:=$ Recall at pixel level.

The figures in Table 1 are the average for 10 trials with different blotch simulations. To compare all the methods in equal basis in each case we report the best results with respect to the F1 score. This score measure the method accuracy averaging with the same weights precision and recall.

The best results of SROD were obtained with the parameter $T=2$. For ASDI, as noted by the authors, we observed little differences for values of parameter $\epsilon$ in the range $\left[10^{-8}, 10^{-2}\right]$. For our proposed method the threshold was set between 1 and 3 with best results obtained for $T d=1$. 
Table 1. Evalaution Scores for the three evaluated methods. The figures were obtained averaging 10 trials with different blotch simulations.

\begin{tabular}{|c|c|c|c|}
\hline & SROD & $\overline{\text { Proposed }}$ & ASDI \\
\hline FNp & 323 & 122 & 119 \\
\hline FPp & 5 & 86 & 1447 \\
\hline FN & 0 & 0 & 0 \\
\hline FP & 0 & 1 & 544 \\
\hline $\mathrm{P}$ & 0,930 & 0,894 & 0,397 \\
\hline $\mathrm{R}$ & 0,744 & 0,911 & 0,914 \\
\hline F1 & 0,850 & 0,886 & 0,532 \\
\hline
\end{tabular}

In all cases the motion estimation and compensation was performed with the Lucas-Kanade approach [5]. We used a pyramidal implementation developed by Bouguet [1].

\subsection{Results}

From the results summarized in Table 1 we can conclude the following. No method outperforms the other two in all scores. The proposed method is the one with best balance between FPp and FNp and precision very close to the one obtained by ASDI. This leads to the best F1 score among the three evaluated methods. On the other hand, the reduction of FNp comes at the expense of increasing the number of FPp. For this reason SROD gives the best result in terms of FPp but the worst in terms of FNp. ASDI suffers from a large number of FPp and FP due to the detection of a large collection of small false blotches. The FP in our method correspond to a false detection due to an error in the motion estimation-compensation step, see Figure 4.3 .

Now we are going to discuss the results from the point of view of the final application. While performing film restoration we want a low number of FP. If the restoration will be assisted by a technician it is very cumbersome to have a large number of false positive. If the restoration is to some extent automatic, a large number of false positive blotches may produce the modification of the frame in too many places increasing the chances of observing the corrections in the restored film. Also, this will produce an increase in the computation cost of the restoration. Based on these considerations the best two methods are SROD and the proposed one. ASI should be improved to reduce the large number of FPp and FP. We believe this can be implemented using a post-processing step.

Regarding the quality of the blotch detection, a useful method should produce the smallest number of FNp. In this way, the restoration after detection is simplified and the chances of an undetectable restoration are increased. FNp usually concentrate at the borders of the blotches which may deteriorate the restorations steps which usually involve some kind of inpanting procedure. From this point of view the best methods are ASDI and the proposed one. 
If we take all the previous comments into consideration we conclude the following. Although none of the methods clearly outperforms the other two, the proposed method is the one with highest $\mathrm{F} 1$ score while achieving a FNp rate close to the best obtained by ASDI. Furthermore, the FPp and FP scores of the proposed method are clearly better than ASDI. When comparing our method with SROD we can see that we reduced the number of FNp increasing the number of FPp. It must be noted that this increase is due to only one blotch (FP) produced by our method. Hence, from the application point of view our method is a good balance between SROD and ASDI. Since, all methods have their strong and weak points a combination of them could lead to a better performance. This will be part of our future work.

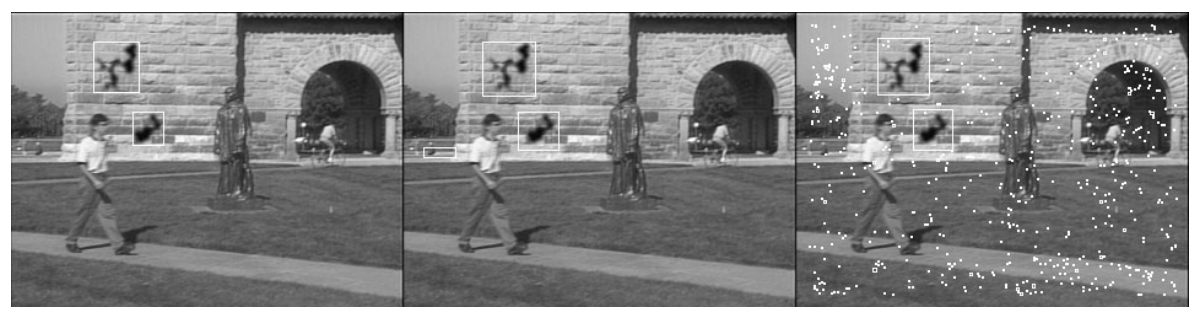

Fig. 1. Left: Results with SROD. Middle: Proposed Method. Right: ASDI.

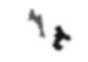

Fig. 2. Sample of simmulated blotches

\section{Conclusions}

In this paper we addressed the problem of blotch detection in the context of digital film restoration. A new method for blotch detection together with an objective evaluation methodology of the detection performance was proposed. Based on this objective methodology we compared our proposed method for blotch detection with two other references from the literature, a classical method [7] and a recently introduced one [2]. We showed that the proposed method outperforms in some of the scores proposed the other two. Also, we argued that from an application point of view our proposed method has clear benefits. Finally, since none of the three methods outperforms the other two in all scores we believe there is room for improvement via the combination of the three methods. This will be part of our future work. 


\section{References}

1. Bouguet: Pyramidal implementation of the lucas kanade feature tracker description of the algorithm. Technical report, Intel Corporation (2002)

2. Buades, A., Delon, J., Gousseau, Y., Masnou, S.: Adaptive blotches detection for film restoraion. In: ICIP 2010, pp. 3317-3320 (2010)

3. Desolneux, A., Moisan, L., Morel, J.M.: Gestalt Theory and Image Analysis. Springer, Heidelberg (2007)

4. Kokaram, A.: Motion Picture Restoration: Digital Algorithms for Artefact Suppression in Degraded Motion Picture Film and Video. Springer, Heidelberg (2001)

5. Lucas, B., Kanade, T.: An iterative image registration technique with an application to stereo vision. In: Image Understanding Workshop, pp. 674-679 (1981)

6. Pinz, A., Schallauer, P., Haas, W.: Automatic restoration algorithms for $35 \mathrm{~mm}$ film. J. Computer Vision Research 1(3), 59-85 (1999)

7. Van Roosmalen, P.M.B.: Restoration of Archvied Film and Video. PhD thesis, Delft University (1999) 